\title{
Novel approaches targeting mitochondrial fission to deplete stem-like tumor cells in prostate cancer and improve treatment outcomes
}

\author{
Antonino Passaniti ${ }^{1,2,3,4}$, Arif Hussain ${ }^{1,2,3,4}$ \\ ${ }^{1}$ Department of Pathology, ${ }^{2}$ Department of Biochemistry \& Molecular Biology, ${ }^{3}$ The Marlene \& Stewart Greenebaum Comprehensive Cancer \\ Center, University of Maryland School of Medicine, Baltimore, MD, USA; ${ }^{4}$ The Veteran's Health Administration Research \& Development Service, \\ Baltimore, MD, USA \\ Correspondence to: Antonino Passaniti, PhD. Department of Pathology, Greenebaum Comprehensive Cancer Center, University of Maryland School \\ of Medicine, 655 W Baltimore Street, BRB 9-045, Baltimore, MD 21201, USA. Email: tpassaniti@som.umaryland.edu. \\ Provenance: This is an invited article commissioned by the Section Editor Dr. Xiao Li, MD (Department of Urology, Jiangsu Cancer Hospital \& \\ Jiangsu Institute of Cancer Research \& Nanjing Medical University Affiliated Cancer Hospital, Nanjing, China). \\ Comment on: Civenni G, Bosotti R, Timpanaro A, et al. Epigenetic Control of Mitochondrial Fission Enables Self-Renewal of Stem-like Tumor Cells \\ in Human Prostate Cancer. Cell Metab 2019;30:303-18.e6.
}

Submitted Sep 06, 2019. Accepted for publication Sep 18, 2019.

doi: 10.21037/atm.2019.09.109

View this article at: http://dx.doi.org/10.21037/atm.2019.09.109

\section{Advanced prostate cancer and the impact of treatments}

Prostate cancer is the most commonly diagnosed cancer in men in the United States with 165,000 new cases diagnosed in 2018 (1). It is the second leading cause of cancer mortality in men, with almost 30,000 deaths in 2018 attributed to progressive metastatic disease. Although initially responsive to hormonal therapies [such as androgen deprivation therapy (ADT) or suppression of gonadalderived testosterone], men invariably progress and develop castrate resistant prostate cancer (CRPC), a disease state that represents progressive disease despite castrate levels of serum testosterone. Additional responses may occur in patients with CRPC with secondary hormonal therapies that target androgen biosynthesis and/or the androgen receptor-the so-called AR axis targeting agents (ARATs). However, these treatments also fail only after a few months in most patients (2-4). Other treatment options for CRPC include chemotherapy, vaccine based therapy (sipuleucel-T), and bone directed therapies such as radiopharmaceuticals, but again these therapies improve overall survival by only a few months (5-7). More recently, based on several large randomized clinical trials that showed a survival advantage particularly for patients with more aggressive higher 'volume' disease, the treatment paradigm for metastatic castration sensitive prostate cancer (CSPC) has begun to shift so that chemotherapy or ARATs are being integrated with ADT upfront in the CSPC setting (8-11). Nevertheless, none of these approaches is curative, and ultimately the majority of men with metastatic prostate cancer succumb to their disease.

Prostate cancer is characterized by significant heterogeneity, both in terms of its underlying biology and clinical course. Although most patients with advanced prostate cancer continue to express AR even in the setting of castration resistance, other phenotypes can emerge, particularly as the disease progresses, including a disease state characterized by neuroendocrine features or even a clinical phenotype that is both AR negative and lacks neuroendocrine characteristics-a subset of which may be driven by FGF, among other factors (12). What drives biological heterogeneity, treatment failure after initial response, de novo treatment resistance, and/or emergence of altered phenotypes upon disease progression are areas of intense investigation. Stem-like tumor cells [i.e., the so-called cancer stem cell (CSC)], which make up a very small proportion of the bulk tumor cell population, have been implicated in some of these processes, including intratumoral heterogeneity and treatment failure. The CSC 
can undergo asymmetric cell division to maintain its own self renewal and also give rise to other daughter cells that become progenitors of the bulk tumor cell population (13). CSC expansion is the result of an imbalance in selfrenewal, differentiation, and senescence pathways. The working hypothesis is that once the proliferative potential of CSCs is lost, the stem cell pool cannot be replenished and cannot contribute to metastasis and drug resistance. Therefore, defining how the CSC phenotype is maintained could provide new clues to treatment for various cancers, including prostate cancer.

\section{Reprogrammed tumor metabolism in prostate cancer}

One of the regulatory pathways for CSC expansion that has recently been considered is the ability of these cells to reprogram their nutrient metabolism. Metabolism in the normal prostate epithelium is unusual in that oxidative phosphorylation is blocked due to high accumulation of zinc in the prostate via zinc transporters that inhibits $\mathrm{m}$-aconitase, which mediates the citrate to isocitrate step of the Krebs cycle. Thus, the normal prostate epithelium accumulates the highest levels of citrate of any cell or organ in the body (14). With malignant transformation, down-regulation of the zinc transporter leads to less zinc uptake and release of m-aconitase inhibition, which results in restoration of oxidative phosphorylation. However, subsequent mutational changes in the prostate epithelial genome may occur and promote a metabolic switch from oxidative phosphorylation to glycolysis that has classically been referred to as the Warburg effect, originally described in the 1920s as one of the hallmarks of malignancy (15). Prostate tumor cells that undergo this transformation may have functional mitochondria but the proliferative phase of the tumor is highly androgen- and glycolyticdependent. With further progression of the disease, metabolic reprogramming may lead to dependence again on mitochondrial oxidative phosphorylation.

\section{Mitochondrial dynamics and CSC}

Regardless of the specific underlying biochemical pathways, a key mechanism that controls mitochondrial metabolism is fission (16). Both mitochondrial biogenesis and mitochondrial fission are integral components of cell growth and division, and can modulate mitochondrial metabolism. Through a series of elegant and detailed cell culture and animal based studies, Civenni et al. now report in a paper in the July 2019 issue of Cell Metabolism entitled "Epigenetic Control of Mitochondrial Fission Enables Self-Renewal of Stem-like Tumor Cells in Human Prostate Cancer" on the mechanisms regulating mitochondrial fission that in turn modulate CSC and tumor cell dynamics (16). Mitochondrial fission is the process of fragmentation that occurs during mitosis, which normally leads to equal partition of mitochondria into daughter cells. In stem cells, including CSC, asymmetric cell division results in daughter cells with different fates (e.g., stem cell self-renewal and generation of other daughter cells with proliferative or differentiation capacities). Mitochondrial fission is a fundamental event in CSCs during their asymmetric division and determines their fate between self-renewal and cell exhaustion/senescence. Changes in mitochondrial size can often influence the rate of mitochondrial repositioning on the cortical cytoskeleton and thus regulate asymmetrical partitioning of mitochondria along the nuclear membrane (17). For stem cells, dysfunctional mitochondria may be eliminated via autophagy or senescence, which may prevent stem cell exhaustion. It is believed that targeting the factors that regulate mitochondrial fission in CSCs could preferentially affect stem-like cells. Bromodomain and extra-terminal domain (BET) proteins, such as BRD4, play a role in cell typespecific (perhaps CSC-like) transcriptional programs and mitochondrial fission, as now reported in prostate cancer CSC (16).

\section{BET proteins and CSC}

It is well established that the BET protein BRD4 can interact with $\mathrm{AR}$ to regulate $\mathrm{AR}$-dependent transcription and proliferation of prostate cancer cells (16). For instance, the novel BET inhibitor (OTX015), which is undergoing clinical development for hematopoietic and prostate tumors, can interfere with protein: protein interactions and block the transcriptional program in AR-dependent prostate cancer cells, thus inhibiting the expression of target genes. More specifically, using gene knockdown and OTX015 inhibitor treatments and rescue experiments with ectopic BRD4 and MFF expression, new results provide evidence for the role of BET proteins in regulating mitochondrial fission in both AR-positive and AR-negative androgen sensitive and androgen insensitive prostate cancer cells, particularly in the small stem-like tumor (CSC) subpopulation that exists within the tumor bulk. It was found that maintenance 
of mitochondrial morphology and function is important in sustaining self-renewal and tumorigenic capacity of prostate CSCs and that BRD4 plays a role in regulating the expression of the mitochondrial fission factor (MFF) to promote mitochondrial fission and sustained CSC-like expansion of a subset of bulk prostate tumor cells that may be important in metastasis and treatment resistance.

\section{Key findings of the study}

This new study of epigenetic control revealed two important results: (I) a role for mitochondrial fission in CSC-like expansion; (II) the potential impact of these findings on clinical prostate cancer treatment. The effect of genetic depletion of BRD4 was compared with the effect of a chemical BET inhibitor (OTX015) on CSC-like tumor cells in several prostate cancer cell lines.

\section{CSC growth characteristic and gene expression patterns}

It was found that a common CSC population from either AR-dependent or AR-independent tumor cells appears to be sensitive to the BET inhibitor (OTX015) using bulk proliferation (2D culture) vs. tumorsphere grown (3D culture; CSC-like) cell lines. Colony forming assays (CFA) and $e x$ vivo cell culture (from xenografts implanted in immune-compromised mice) were used to confirm these results. It was concluded that OTX015 treatment more selectively targets CSC-like cells than non-CSC-like cells, especially as shown in the tumorsphere assays. To test whether BET proteins are required to sustain the CSC-like component of prostate cancer tumors, the effects of shRNA targeting BRD4 (knockdown) were assessed via CFA, proliferation in 2D culture, tumorspheres (3D culture), and tumor growth in vivo. The possible involvement of senescence in CSC-like depletion was validated using senescent-associated $\beta$-Galactosidase as a marker (SA- $\beta \mathrm{Gal})$. BRD4 knockdown showed that CSC-selective inhibition appears to be mediated through increased senescence (SA$\beta G a l)$, which may inhibit self-renewal and/or decrease the CSC-like population. BRD4 knockdown showed essentially similar activity as OTX015 drug treatment. Gene expression analysis in 2D culture cells showed a downregulation of proliferation markers while cells cultured in $3 \mathrm{D}$ tumorspheres showed a downregulation of metabolism markers. One important result of the study is that spatial organization alters gene expression and signaling pathways in radically different ways. In tumorsphere (CSC-like) cells, expression of metabolism and mitochondrial pathways that are sensitive to drugs targeting mitochondrial fission are altered but not in cells grown in 2D. Therefore, inhibiting BET has consequences for specific transcriptional programs in prostate CSC-like cells rather than bulk culture tumor cells.

\section{Mitochondrial dynamics and metabolism}

If suspension (3D) culture favors CSC-like cells, mitochondrial morphology and/or function within the $3 \mathrm{D}$ context may be different than in the bulk population. Indeed, it was found that abnormal mitochondrial morphology and dysfunctional mitochondria were observed after OTX015 or BRD4 knockdown, specifically in cells grown in suspension 3D cultures. Using Mitotracker Green to track mitochondrial morphology and Mitotracker Red, TOM20 antibody, and TMRM to track function, it was clear that BET inhibition alters both mitochondrial dynamics and morphology. BRD4 knockdown or BET inhibition also disrupted mitochondrial membrane potential (MMP), lowered oxygen consumption rates (OCR), and inhibited spare respiratory capacity (SRC). To measure MMP, the mitochondrial-selective dye JC1 was used in fluorescence-activated cell sorting protocols, while the seahorse XF-extracellular flux analyzer was used to measure respiratory efficiency. This critical mitochondrial parameter is expressed as maximal respiratory capacity (MRC) by subtracting non-mitochondrial respiration (NM; OCR value after adding Antimycin A) from the FCCP-inducible rate (MRC). ATP production-linked respiration (AP) is calculated after subtracting the oligomycin rate (inhibits all mitochondrial OCR) from baseline cellular OCR. Proton leak-linked respiration (PL) is defined as the difference between the oligomycin rate and NM (subtracting NM from the oligomycin rate). Importantly, SRC is obtained after subtracting basal respiration (OCR value at $\mathrm{t}=12 \mathrm{~min}$ ) from MRC while baseline cellular OCR (BL) is calculated from basal respiration after subtracting NM. Respiratory capacity, oxygen consumption rates, and MMP were all affected by BET inhibition and these changes were associated with hyper-fused mitochondria and loss of selfrenewal capacity.

\section{Mitochondrial fission}

An important target gene that is upregulated by BRD4 and binds to a receptor (DRP1) that regulates mitochondrial 
fission is the MFF. It was found that MFF and BRD4 protein expression correlated using immunohistochemistry in hormone-naïve primary and hormone-refractory metastatic human prostate tumors. Colocalization of MFF and BRD4 was also observed in cultured cells. Expression (mRNA levels) of MFF in adherent (2D) or suspension (tumorsphere) cells was inhibited by OTX015 and MMF promoter occupancy was also downregulated, as demonstrated by chromatin immunoprecipitation (ChIP)Seq assays. Co-expression of BRD4 and MFF in primary and metastatic tumors and co-localization in mitochondria of cultured cells suggests a conserved function, while OTX015 inhibition of MFF expression at the mRNA level suggests that BRD4 transcriptionally activates MFF, the MFF. MFF also appeared to regulate mitochondrial fission and CSC-like tumorigenesis of prostate cancer cells. Tumorsphere versus bulk proliferation and growth of xenografts of MFF-depleted DU145 and PC3 prostate cancer cells were measured using ex vivo tumorsphere forming ability of xenografts isolated from in vivo DU145 and PC3 tumors with or without MFF knockdown. Mitochondrial morphology, as assessed by TOM20 immunostaining in adherent and tumorsphere DU145 cells after MFF knockdown with shRNAs, was altered. MMP (after MFF knockdown), OCR, SRC, and senescence were all rescued by ectopic expression of MFF. Ectopic expression of MFF also rescued OTX105-inhibited tumorspheres. Mitochondrial morphology and dysfunction were recovered with ectopic MFF (after OTX015 treatment). Taken together, the combined data suggest that MFF overexpression mediates the BRD4-activated mitochondrial fission program. It is likely that MFF regulates stem-like and tumorigenic properties of prostate CSCs.

\section{Conclusions and summary}

The authors conclude that prostate CSCs appear to depend on mitochondrial fission to maintain self-renewal capacity. BRD4 inhibition lowered MFF to block mitochondrial fission and clearance of defective mitochondria, inhibiting CSC-like tumor cell growth and tumorigenesis (16). Therefore, BRD4 could mediate a CSC-like transcriptional program that promotes self-renewal and expansion of prostate cancer CSCs. The BRD4-regulated transcriptional program (through MFF) and mitochondrial function could potentially be useful targets to inhibit CSC expansion and to promote exhaustion. In this study, AR-independent effects of BRD4 are revealed for the first time and it is possible that targeting CSC self-renewal by blocking mitochondrial reprogrammed fission events (BET inhibitors) could be exploited for therapeutic benefit, including possibly for both castration sensitive and castration resistant prostate cancers. The authors acknowledge that BRD4 may regulate other mitochondrial and metabolic pathways in prostate CSCs and whether this has direct consequences for prostate tumorigenesis remains to be elucidated. Other experimental models (primary human cells, organoids, or patient-derived xenografts) and analysis of additional human tumor samples would also be relevant to revealing the basic mechanisms of mitochondrial fission that control CSC expansion, which may be applicable to other tumor types as well. However, it is not clear from this study why MFF control of mitochondrial fission bestows a survival advantage on CSCs but not the bulk tumor. That is, what is the source of mitochondrial function in asymmetric cell division? Are there epigenetic (or mutational) changes in mitochondrial DNA that play a role or do metabolic enzymes and nutrients play different (non-canonical) roles under conditions of asymmetric division associated with MFFdriven mitochondrial fission? The answers to these and other questions could clarify specific additional mechanisms through which BET-regulated mitochondrial fission might control CSC fate and prostate cancer metastasis, further increasing the clinical impact of the findings from this important study.

\section{Acknowledgments}

The authors would like to thank Drs. Myoung Sook Kim and Minhaj Siddiqui (University of Maryland Comprehensive Cancer Center) for helpful discussions and advice.

Funding: A Passaniti [NIH/NCI 2P30CA134274-09 NIH (NCI) Program project grant; 1R43CA224750 NIH SBIR grant]; A Hussain [VA Merit Award (I01 BX000545, Medical Research Service, Dept of Veterans Affairs); NIH/ NCI 2P30CA134274-09 NIH (NCI) Program project grant].

\section{Footnote}

Conflicts of Interest: The authors have no conflicts of interest to declare.

Ethical Statement: The authors are accountable for all aspects of the work in ensuring that questions related 
to the accuracy or integrity of any part of the work are appropriately investigated and resolved.

\section{References}

1. Siegel RL, Miller KD, Jemal A. Cancer statistics, 2018. CA Cancer J Clin 2018;68:7-30.

2. Merseburger AS, Haas GP, von Klot CA. An update on enzalutamide in the treatment of prostate cancer. Ther Adv Urol 2015;7:9-21.

3. de Bono JS, Logothetis CJ, Molina A, et al. Abiraterone and increased survival in metastatic prostate cancer. $\mathrm{N}$ Engl J Med 2011;364:1995-2005.

4. Ryan CJ, Smith MR, Fizazi K, et al. Abiraterone acetate plus prednisone versus placebo plus prednisone in chemotherapy-naive men with metastatic castrationresistant prostate cancer (COU-AA-302): final overall survival analysis of a randomised, double-blind, placebocontrolled phase 3 study. Lancet Oncol 2015;16:152-60.

5. Oudard S, Fizazi K, Sengeløv L, et al. Cabazitaxel Versus Docetaxel As First-Line Therapy for Patients With Metastatic Castration-Resistant Prostate Cancer: A Randomized Phase III Trial-FIRSTANA. J Clin Oncol 2017;35:3189-97.

6. Kantoff PW, Higano CS, Shore ND, et al. Sipuleucel-T immunotherapy for castration-resistant prostate cancer. $\mathrm{N}$ Engl J Med 2010;363:411-22.

7. Parker C, Nilsson S, Heinrich D, et al. Alpha emitter radium-223 and survival in metastatic prostate cancer. $\mathrm{N}$ Engl J Med 2013;369:213-23.

8. Sweeney CJ, Chen YH, Carducci M, et al.

Cite this article as: Passaniti A, Hussain A. Novel approaches targeting mitochondrial fission to deplete stem-like tumor cells in prostate cancer and improve treatment outcomes. Ann Transl Med 2019;7(Suppl 8):S335. doi: 10.21037/atm.2019.09.109
Chemohormonal Therapy in Metastatic HormoneSensitive Prostate Cancer. N Engl J Med 2015;373:737-46.

9. Fizazi K, Tran N, Fein L, et al. Abiraterone plus Prednisone in Metastatic, Castration-Sensitive Prostate Cancer. N Engl J Med 2017;377:352-60.

10. Davis ID, Martin AJ, Stockler MR, et al. Enzalutamide with Standard First-Line Therapy in Metastatic Prostate Cancer. N Engl J Med 2019;381:121-31.

11. Chi KN, Agarwal N, Bjartell A, et al. Apalutamide for Metastatic, Castration-Sensitive Prostate Cancer. N Engl J Med 2019;381:13-24.

12. Bluemn EG, Coleman IM, Lucas JM, et al. Androgen Receptor Pathway-Independent Prostate Cancer Is Sustained through FGF Signaling. Cancer Cell 2017;32:474-89.e6.

13. Maitland NJ, Collins AT. Prostate cancer stem cells: a new target for therapy. J Clin Oncol 2008;26:2862-70.

14. Franklin RB, Milon B, Feng $\mathrm{P}$, et al. Zinc and zinc transporters in normal prostate and the pathogenesis of prostate cancer. Front Biosci 2005;10:2230-9.

15. DeBerardinis RJ, Chandel NS. Fundamentals of cancer metabolism. Sci Adv 2016;2:e1600200.

16. Civenni G, Bosotti R, Timpanaro A, et al. Epigenetic Control of Mitochondrial Fission Enables Self-Renewal of Stem-like Tumor Cells in Human Prostate Cancer. Cell Metab 2019;30:303-18.e6.

17. Caino MC, Altieri DC. Molecular Pathways: Mitochondrial Reprogramming in Tumor Progression and Therapy. Clin Cancer Res 2016;22:540-5. 